\title{
LA NATURALEZA DEL VERBO DE LAS COLOCACIONES VERBONOMINALES CON 'DAR'
}

\begin{abstract}
Bustos Plaza Alberto, La naturaleza del verbo de las colocaciones verbonominales con 'dar' [The nature of the verbs of the verbo-nominal combinations with 'dar']. Studia Romanica Posnaniensia, Adam Mickiewicz University Press, Poznań, vol. XXXI: 2004, pp. 175-181. ISBN 83232-1353-4, ISSN 0137-2475.

It has often been stated that Spanish combinations such as dar un beso ('to give a kiss'), dar un paseo ('take a walk') etc. contain a grammaticalized verb whose function consists in conjugating an abstract noun. A comparison with the equivalent Latin combinations does not provide any evidence of the hypothesized grammaticalization process. The grammatical status of the verb is not confirmed either by parameters such as Lehmann's $(1985,1995)$. Those are reasons to reconsider the hypothesis of the grammaticalization of this verb.
\end{abstract}

\section{INTRODUCCIÓN}

Son muy frecuentes en español las colocaciones formadas por el verbo dar y un sintagma nominal, como, por ejemplo, dar un beso, dar una noticia, dar un salto, dar un paseo, etc. Este tipo de combinaciones del español y de otras lenguas viene llamando la atención de los lingúistas al menos desde principios del siglo XX. Uno de los puntos en los que se ha centrado el interés es el de la posible gramaticalización del verbo.

Se ha tratado de dar cuenta del verbo de estas combinaciones mediante conceptos como el de verbo formemático (cf. Šabršula, 1966), verbo funcional (cf. para el alemán Polenz, 1963; Helbig, 1984; para el francés Detges, 1996; para el castellano Busch, 1985), verbo de apoyo (cf. Gross, 1989) o verbo liviano (cf. Grimshaw y Mester, 1988). A esta diversidad de denominaciones subyace el intento de explicar un mismo fenómeno desde diferentes tradiciones y perspectivas teóricas. Todos estos conceptos comparten una base común, que podemos resumir como la idea de que estas combinaciones contienen un verbo gramaticalizado cuya 
función, comparable a la de un auxiliar, consiste en conjugar un sustantivo abstracto. Así, la combinación de verbo y sintagma nominal asume, supuestamente, un papel comparable al de una perífrasis verbal. Este planteamiento presupone que el verbo dar de combinaciones como dar un abrazo o dar un paseo ha sufrido un proceso de gramaticalización por el cual ha surgido un verbo semejante a un auxiliar a partir del verbo pleno homófono.

\section{INCONVENIENTES DE LA HIPÓTESIS DE LA GRAMATICALIZACIÓN DEL VERBO}

A nuestro juicio, una objeción que se les puede plantear razonablemente a los diversos trabajos que defienden esta hipótesis es que se conforman con afirmar que se ha producido el proceso de gramaticalización sin documentar su desarrollo. Ahora bien, Laca (1996: 22 s.) previene ante esta forma de actuar -frecuente, por otra parte, en los estudios sobre gramaticalización- y hace hincapié en la necesidad de contrastar con materiales históricos tales hipótesis siempre que se trabaje con lenguas cuya tradición escrita sea lo suficientemente dilatada.

No emprenderemos aquí un estudio histórico del léxico, pues esto rebasaría con creces los límites de este artículo, sino que nos limitaremos a aportar algunos ejemplos latinos que nos permitan contrastar dos estadios sincrónicos diferentes de un mismo fenómeno $\mathrm{y}$, de esta manera, juzgar si se ha producido algún tipo de evolución histórica que arroje luz sobre la hipotética gramaticalización del verbo de las combinaciones verbonominales.

\subsection{COMBINACIONES VERBONOMINALES EN LATÍN}

En latín son numerosas las combinaciones verbonominales con dare equivalentes a las actuales castellanas. Este hecho ya lo pusieron de manifiesto diversos investigadores no solo en relación con dare, sino también con otros verbos (cf. Dubský, 1963: 46; Šabršula, 1961: 100 s.; Thun, 1981: 325, 342-345). Nosotros hemos encontrado abundantes ejemplos de combinaciones latinas con dare comparables con las del castellano actual. He aquí algunos ejemplos tomados de Perseus (Crane, http://www.perseus.tufts.edu/, octubre de 2002):

Anni autem tempus libertatem maiorem mihi dat (Cicerón: Epistulae ad Familiares, libro 10. carta 33, sec. 5, ed. L. C. Purser)

Tum vero ingentem gemitum dat pectore ab imo (Virgilio, Eneida, libro 1, lín. 485, ed. J. B. Greenough)

[...] dat puer oscula matri (Ovidio: Metamorfosis, libro 10, lín. 525, cd. Hugo Magnus) 
Se pueden encontrar otros muchos ejemplos, como animos dare, colloquium dare, poenas dare, facultatem dare, sonitum dare, consilium dare, etc. Algunas de estas combinaciones siguen existiendo actualmente en castellano, como, por ejemplo, libertatem dare, que tiene un descendiente castellano dar libertad. En otros casos, encontramos combinaciones equivalentes por su significado con sustitución de piezas léxicas latinas por sus equivalentes españoles actuales, como osculum dare, cuyo equivalente actual es dar un beso.

Si dejamos de lado las diferencias morfosintácticas, este grupo de combinaciones mantiene su identidad en lo esencial. En concreto, el uso del verbo es idéntico en las combinaciones latinas y en las españolas: ¿qué diferencia hay, por ejemplo, entre el verbo de dar libertad y el de libertatem dare? A nuestro entender, no hay ninguna. Las dos combinaciones significan exactamente lo mismo. Esto no significa necesariamente que el verbo de estas combinaciones no esté gramaticalizado, pues podría ser que ya lo estuviera en latín, con lo que el proceso de gramaticalización se remontaría a una época anterior a la documentación conservada. No obstante, en ese caso, la hipótesis no sería necesariamente falsa, pero sí incontrastable, por lo que quizás fuera más prudente dejarla en suspenso. Ahora bien, la situación que encontramos en latín no excluye una hipótesis alternativa: podría ser que el supuesto proceso de gramaticalización no hubiera tenido lugar, en cuyo caso, el verbo no estaría gramaticalizado.

\subsection{PARÁMETROS DE GRAMATICALIZACIÓN}

Dado que la teoría de la gramaticalización nos brinda instrumentos para comprobar sincrónicamente el carácter gramaticalizado de un signo, aplicaremos estos para tratar de juzgar objetivamente sobre el presunto carácter gramatical del verbo. Nos serviremos aquí de los parámetros de gramaticalización de Lehmann (1985, 1995), que aplicaremos únicamente a las combinaciones castellanas. Si el verbo no está gramaticalizado en estas, es imposible que lo estuviera en latín. Examinamos a continuación los diversos parámetros uno a uno'.

a. Integridad: este parámetro tiene una vertiente fonológica y otra semántica. Cuanto mayor es la reducción de la sustancia fónica y del contenido semántico de un signo dado, mayor es el grado de gramaticalización. El verbo de las combinaciones del tipo dar un abrazo no ha sufrido erosión alguna en su sustancia fónica. Es idéntico, a este respecto, al verbo inequívocamente pleno de dar $10 € \mathrm{Ea}$ disminución de la integridad de un signo lleva asociada una degeneración morfológica que desemboca en la pérdida de la flexión (Lehmann, 1985: 307). Evidentemente, este no es el caso de los verbos que nos ocupan, pues mantienen sin alteración las desinencias verbales.

\footnotetext{
' Para la descripción de los parámetros seguimos a Lehmann (1985, 1995).
} 
No entraremos en el aspecto semántico del problema, ya que esto superaría con creces las posibilidades del presente trabajo; pero, como trataremos de poner de manifiesto, los restantes argumentos indican que el verbo no está gramaticalizado.

b. Paradigmaticidad: por paradigmaticidad se entiende el grado de integración de un signo en un paradigma. Los elementos léxicos forman clases abiertas a las que se pueden sumar nuevos elementos indefinidamente, mientras que el repertorio de elementos gramaticales aumenta de manera muy escasa. Si el verbo dar fuera equivalente a un auxiliar, debería estar integrado en un paradigma restringido. La gran variedad de verbos que aparecen en estas combinaciones bastaría por sí sola para cuestionar una hipotética gramaticalización. Por ejemplo, con golpe y sustantivos relacionados, podemos encontrar, al menos, ocho verbos diferentes: dar, atizar, arrear, asestar, propinar, descargar, encajar y pegar.

$\mathrm{Si}$ le atribuimos condición gramatical al verbo de los ejemplos anteriores, hay que preguntarse acto seguido si se debe hacer extensiva tal condición a los verbos que, con ese mismo grupo de sustantivos, expresan pasividad, como recibir un golpe y sufrir un golpe (cf. Koike, 2001: 91 ss.; Alonso Ramos, 1998: 196 ss.; Busch, 1985: 6.2). Esa misma pregunta resultaría pertinente para el verbo de combinaciones ocasionales que también expresan pasividad, como sentir un golpe y acusar un golpe.

Habría que tener en cuenta también aquellos verbos que expresan valores aspectuales, como repetir un golpe (iterativo), y amagar (conativo) (cf. Koike, 2001: 97 ss.; Alonso Ramos, 1998: 193-196; Busch, 1985). Otros presentan valores más difíciles de etiquetar como parar un golpe, atajar, evitar y esquivar, que indican la evitación del proceso, marrar un golpe, que indica que el proceso no llega a buen término, mitigar y paliar, que indican la atenuación de los efectos del proceso, y dirigir, que se relaciona con el control del proceso por parte del agente.

Según se aprecia, hay una amplia gama de verbos que permite expresar gran cantidad de matices. En realidad, encontramos una proliferación de verbos que no se pueden reducir a paradigmas cerrados. No se le puede atribuir valor gramatical a este conjunto heterogéneo.

c. Variabilidad paradigmática: este parámetro presenta una estrecha correlación con el de paradigmaticidad. La gramaticalización no solo reduce el tamaño de los paradigmas, sino que la elección en el interior de estos viene condicionada progresivamente por reglas gramaticales; es decir, con la gramaticalización aumenta el grado de obligatoriedad del signo. Los elementos léxicos, a diferencia de los signos gramaticalizados, admiten amplias posibilidades de variación. La obligatoriedad es uno de los indicios más importantes de gramaticalización. Los elementos más gramaticalizados o bien son obligatorios o bien alternan dentro de un conjunto muy restringido (cf. Lehmann, 1985: 307). La obligatorificación va acompañada de un incremento de la distribución del signo y de una reducción de las restricciones de selección (cf. Hopper y Traugott, 1993: 5.2). Con el verbo de las combinaciones verbonominales, sucede todo lo contrario. Tradicionalmente, estas combinaciones han llamado la atención, precisamente, por su imprevisibilidad. 
Sustantivos con significados afines, como disparo y tiro, se combinan con verbos diferentes: dar un tiro / realizar un disparo / ?realizar un tiro /?dar un disparo. Es muy reveladora a este respecto la perplejidad de María Moliner al intentar clasificar las combinaciones con dar:

Este verbo tiene multitud de usos, muchos de los cuales son casos intermedios entre modismos y acepciones, ya que con palabras de uso tan semejante que sería imposibie distinguir en una definición, es aplicable en un caso y no lo es en otro. Por ejemplo, se dice 'dar un aviso', pero no 'dar una advertencia'. Sí, 'dar paso'; no, 'dar tránsito'. Sí, 'dar un grito': no, 'dar un trino'. Sí, 'dar la enhorabuena'; no, 'dar una felicitación' [...] (Moliner, 1996: desarrollo gramatical de dar).

d. Alcance estructural: la gramaticalización reduce el alcance estructural de los signos $^{2}$, es decir, como consecuencia de la gramaticalización, un signo dado se va combinando en el curso de su evolución histórica con constituyentes de complejidad decreciente. Por ejemplo, un verbo pleno rige toda una oración, mientras que un elemento gramaticalizado, como un verbo auxiliar (ha corrido) o un morfema (correrá), modifica únicamente a un elemento léxico.

Hay indicios de la condición argumental del sintagma nominal de estas combinaciones; por ejemplo, la posibilidad de focalización mediante una perífrasis de relativo (cf. Moreno Cabrera, 1999):

le di una explicación de los hechos a mi hermano

es una explicación de los hechos lo que le di a mi hermano

Si el sintagma nominal es un argumento del verbo, la situación es idéntica a la de combinaciones como dar $10 \epsilon$, en las que el verbo es predicativo. El verbo de las combinaciones verbonominales no es, por tanto, un auxiliar que modifique al sintagma nominal, sino que es un predicado que convierte a este último en su argumento. Es decir, el verbo no tiene condición gramatical.

e. Ligazón: por ligazón se entiende el grado de fusión con otros signos. A medida que progresa la gramaticalización, el signo se va desplazando a lo largo de una escala que va de mayor a menor independencia, con diversos estadios, como la yuxtaposición, la cliticización, la aglutinación y, finalmente, la fusión (cf. Hopper y Traugott, 1993: 5.3). El verbo de las combinaciones del tipo dar un abrazo goza de independencia gráfica y fónica: se escribe separado y mantiene su propio acento. No se diferencia en esto lo más mínimo del verbo de combinaciones como dar $10 €$,

${ }^{2}$ Este parámetro ha sido objeto de algunas críticas. Diewald (1997: 23) presenta dos objeciones: a) la denominación alcance se presta a confusión con otros usos; b) con la gramaticalización, puede aumentar el alcance de un signo. La autora cita como ejemplo ta conjunción subordinante alemana dass, que surge del demostrativo das. El demostrativo se sitúa en el interior de la estructura de constituyentes de la oración en la que aparece. En cambio, la conjunción une la totalidad de la oración subordinada con la oración principal. Tabor y Traugott (1998) examinan diversos casos en los que el proceso de gramaticalización lleva aparejado un aumento de alcance estructural. 
que es claramente un verbo pleno con función predicativa. Por tanto, tampoco este parámetro habla a favor de la hipotética gramaticalización.

f. Variabilidad sintagmática: este parámetro tiene que ver con la libertad de desplazamiento en el eje sintagmático. En un primer estadio, el signo gramaticalizado tiende a ocupar una posición sintáctica fija. Posteriormente, puede pasar a ocupar una posición morfológica fija. El verbo y el sintagma nominal de las combinaciones con dar gozan de gran libertad en cuanto a su posición relativa:

Si tanto asco te da el vino, no deberías beber

Temí dar en algún momento signos de confusión

Como vemos en los ejemplos anteriores, el sintagma nominal puede aparecer en ocasiones antepuesto al verbo y se pueden intercalar también otros elementos entre ambos. Así pues, este parámetro también contradice la hipótesis de la gramaticalización del verbo.

En resumen, los parámetros examinados no aportan pruebas de la gramaticalización del verbo de las combinaciones del tipo dar un abrazo, sino que, por el contrario se pueden aportar como evidencia de que este no está gramaticalizado.

\section{CONCLUSIÓN}

No hemos encontrado pruebas históricas de la hipotética gramaticalización del verbo de las combinaciones verbonominales formadas con dar. La situación que encontramos en latín es idéntica a la que se da hoy en castellano. La aplicación de los parámetros de gramaticalización de Lehmann $(1985,1995)$ también contradice la idea de que el verbo esté gramaticalizado. A la vista de estos resultados, debería cuestionarse la hipótesis de la gramaticalización del verbo de estas combinaciones, dado que presenta, más bien, características comparables a las del verbo pleno de combinaciones como dar $10 \epsilon$.

\section{REFERENCIAS BIBLIOGRÁFICAS}

Alonso Ramos, M. (1998), Étude sémantico-syntaxique des constructions à verbe support. Universidad de Montréal, tesis doctoral.

Busch, H.-J. (1985), Zur Bestimmung von Leistungen und Bedeutung ausgewählter Funktionsverbgefüge mit den Verben DAR, HACER, TENER, PONER EN, ENTRAR EN und ESTAR EN im Spanischen. Leipzig, tesis doctoral.

Crane, G. R. (cd.), The Perseus Project. http://www.pcrscus.tufts.edu, octubre de 2002.

Detges, U. (1996), Nominalprädikate: eine valenztheoretische Untersuchung der französischen Funktionsverbgefüge des Paradigmas «être Präposition Nomen» und verwandter Konstruktionen. Tübingen: Niemeyer. 
Diewald, G. (1997), Grammatikalisierung. Eine Einfihhrung in Sein und Werden grammatischer Formen. Tübingen: Niemeyer.

Dubský, J. (1963), Formas descompuestas en el español antiguo. Revista de Filología Española, 46, $31-48$.

Grimshaw, J. y A. Mcster (1988), Light verbs and theta-marking. Linguistic Inquiry, 19/2, 205-232.

Gross, G. (1989), Les constructions converses du français. Genève: Droz.

Helbig. G. (1984), Probleme der Beschreibung von Funktionsverbgefuigen im Deutschen, en Hclbig, G. (ed.), Studien zur deutschen Syntax. Leipzig: Enzyklopädie, t. 2, 163-188.

Hopper, P. J. y E. C. Traugott (1993), Grammaticalization. Cambridge: Cambridge University Press.

Koike, K. (2001), Colocaciones léxicas en el español actual. Estudio formal y léxico-semántico. Alcalá de Henares: Universidad de Alcalá/Takushoku University.

Laca, B. (1996), Methodische Probleme bei der Erforschung von Grammatikalisierungsphänomenen, en Michaelis, S. y P. Thiele (eds.), Grammatikalisierung in der Romania, Bochum: Brockmeyer.

Lehmann, Ch. (1985), Grammaticalization. Synchronic variation and semantic change. Lingua e Stile, 3, 303-318.

Lch man n, Ch. (1995), Thoughts on grammaticalization. München-Newcastle: Lincom.

Moliner, M. (1996), Diccionario de uso del español. Madrid: Gredos, edición en CD-Rom.

Moreno Cabrera, J. C. (1999), Las funciones informativas: las perifrasis de relativo y orras construcciones perifrásticas, en BOSQUE, Ignacio y Violcta Demonte (dirs.), Gramática descriptiva de la lengua española. Madrid: Espasa, vol. 3, 4245-4302.

Polenz, P. (1963), Funktionsverben im heutigen Deutsch. Sprache in der rationalisierten Welt, Wirkendes Wort, Beiheft 5. Düsseldorf.

Sabršula, J. J. (1961), Les locutions semelfactives et l'aspect perfectif en français. Romanistica Pragensia, 2, 99-111.

Šabršula, J. J. (1966), Un problème de la périphérie du système morphologique. À propos des formations prémorphologiques, en Travaux linguistiques de Prague, 2: les problèmes du centre et de la périphérie du système de la langue. Praha-Paris: Academia/Klincksieck, 183-192.

Tabor, W. y E. C. Traugotl (1998), Structural scope expansion and grammaticalization, en Giacalone Ramat, A. y P. Hopper (eds.) (1998), The limits of grammaticalization. Amsterdam-Philadelphia: Benjamins, 229-272.

Thun, H. (1981), Faire école, en Geckeler, H. y otros (eds.). Logos semantikos: Studia linguistica in honorem Eugenio Coseriu (1921-1981). Madrid-Berlin: Gredos/De Gruyter, vol. 111, 325-346. 